\title{
Hybrid model of Monte Carlo simulation and diffusion theory for light reflectance by turbid media
}

\author{
Lihong Wang and Steven L. Jacques \\ Laser Biology Research Laboratory, Box 17, University of Texas M. D. Anderson Cancer Center, \\ 1515 Holcome Boulevard, Houston, Texas 77030
}

\begin{abstract}
Received September 8, 1992; revised manuscript received January 8, 1993; accepted February 23, 1993
Light reflectance by semi-infinite turbid media is modeled by a hybrid of Monte Carlo simulation and diffusion theory, which combines the accuracy of Monte Carlo simulation near the source and the speed of diffusion theory distant from the source. For example, when the turbid medium has the following optical propertiesabsorption coefficient $1 \mathrm{~cm}^{-1}$, scattering coefficient $100 \mathrm{~cm}^{-1}$, anisotropy 0.9 , and refractive-index-matched boundary - the hybrid simulation is 7 times faster than the pure Monte Carlo simulation $(100,000$ photon packets were traced), and the difference between the two simulations is within 2 standard deviations of the Monte Carlo simulation.
\end{abstract}

\section{INTRODUCTION}

In the field of laser-tissue interaction there is a growing demand for accurate and fast models to predict theoretically the light distribution in turbid media, such as biological tissue, that have given optical properties ${ }^{1-3}$ and to deduce inversely the optical properties from measurable quantities. $^{4-6}$ One of the measurable quantities is diffuse reflectance as a function of $r, R_{d}(r)$, where $r$ is the distance between the observation and the incident points of the laser beam on the medium surface and where diffuse reflectance, in this paper, is defined as the photon probability of escape from inside a semi-infinite turbid medium per unit surface area regardless of whether the photon source is inside or outside. Measurement of reflectance can be used to determine the optical properties of tissue noninvasively. ${ }^{5-7}$ Therefore an efficient and accurate model is needed to relate the reflectance and optical properties of a turbid medium.

Monte Carlo simulation ${ }^{8-10}$ offers a flexible and accurate approach toward photon transport in turbid media. It can deal with complex geometries in a straightforward manner and can score multiple physical quantities simultaneously. The accuracy of Monte Carlo simulation has been tested experimentally. ${ }^{11}$ In this paper, Monte Carlo simulation results are used as standards to be compared with hybrid simulation results. However, because of its statistical nature, Monte Carlo simulation usually requires that a large number of photons be traced for acceptable variance to be obtained; hence it is computationally expensive, especially when the absorption coefficient is much less than the scattering coefficient of the medium, in which photons may propagate over a long distance before being absorbed.

Although diffusion theories ${ }^{5,7}$ offer a fast approach to approximating certain physical quantities of light transport in turbid media, they are not valid near the photon source or the boundary, where the photon intensity is strongly anisotropic, thus violating the assumption of diffusion theory. In therapeutic applications of lasers in medicine, the photon fluence near the source is the site of the most intense laser-tissue interaction. This region is where diffusion theory is most inaccurate. In diagnostic and dosimetric measurements, such as the diffuse reflectance $R_{d}(r)$, the reflectance near the source is the strongest and therefore can be more easily and more accurately measured experimentally. Again, this region is where diffusion theory is most inaccurate.

In this paper we describe a hybrid model of Monte Carlo simulation and diffusion theory. The hybrid model computes the diffuse reflectance of an infinitely narrow photon beam normally incident upon a semi-infinite homogeneous turbid medium with given optical properties. Although no photon beam is infinitely narrow, its response can be convolved to compute the response of a finite-sized photon beam. ${ }^{9}$ Although the real tissue can never be infinitely wide, it can be so treated on the condition that it is much wider than the spatial extent of the photon distribution.

Monte Carlo simulation, while collecting some reflectance $R_{m c}(r)$ that is due to near-surface scatterings, is used initially to propagate photons to sufficient depth into the turbid media that diffusion theory can be applied with good accuracy. Diffusion theory is then used to compute the reflectance $R_{\text {diff }}(r)$ that is due to the distributed source provided by the Monte Carlo step. The final reflectance $R_{d}(r)$ will be the sum of the two reflectances. The hybrid model combines the accuracy advantage of Monte Carlo simulation and the speed advantage of diffusion theory and is faster than pure Monte Carlo simulation and more accurate than pure diffusion theory.

Flock et al. ${ }^{12}$ presented a conceptually different hybrid model in which a hybrid coupling function is determined by a Monte Carlo calculation for each set of optical and geometrical parameters. This coupling function is then used to correct the computational results of diffusion theory.

\section{THEORY}

The optical properties of a semi-infinite turbid medium can be described with the use of four parameters: rela- 


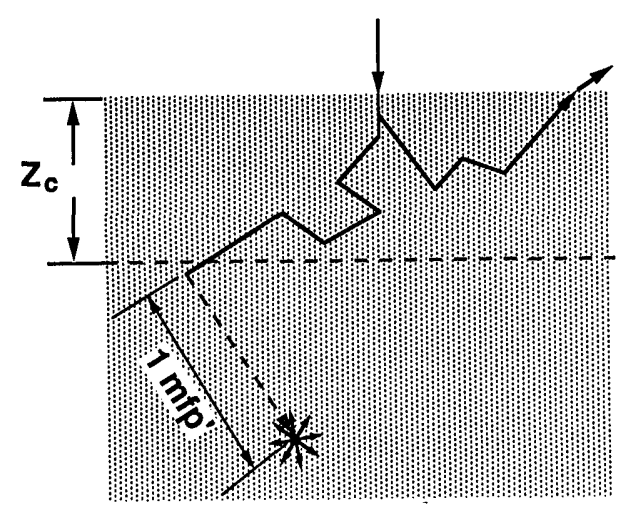

Fig. 1. Illustration of the hybrid model. $Z_{c}$ is the critical depth. The last step size is $1 \mathrm{mfp}^{\prime}$.

tive refractive index $n_{\text {rel }}$, absorption coefficient $\mu_{a}$, scattering coefficient $\mu_{s}$, and the anisotropy factor $g$. The relative refractive index $n_{\text {rel }}$ is the ratio between the refractive indices of the turbid medium and of the ambient medium. The absorption coefficient $\mu_{a}$ is defined as the probability of photon absorption per unit infinitesimal pathlength, and the scattering coefficient $\mu_{s}$ is defined as the probability of photon scattering per unit infinitesimal pathlength. The anisotropy factor $g$ is the average cosine of the scattered angle, where the scattering of tissue is well represented ${ }^{13}$ by a Henyey-Greenstein scattering function. ${ }^{14}$

A cylindrical coordinate system is set up for this problem. The origin of the coordinate system is the point of photon incidence on the medium surface, and the $z$ axis points downward into the turbid medium. The radial coordinate and the azimuthal angle are denoted by $r$ and $\theta$, respectively.

A separate study of diffusion theory ${ }^{7}$ and similarity relations $\mathrm{s}^{15,16}$ conducted by our group (as yet unpublished) has shown that the keys to using diffusion theory accurately are the accurate conversion of the incident infinitely narrow photon beam into deep $\left\{\geq 1 \mathrm{mfp}^{\prime}\right.$, where $\left.1 \mathrm{mfp}^{\prime}=1 /\left[\mu_{a}+\mu_{s}(1-g)\right]\right\}$ isotropic photon sources in the medium and the fact that similarity relations can be applied with good accuracy for deep photons ( $\geq 1 \mathrm{mfp}^{\prime}$ ). Because of its high accuracy, Monte Carlo simulation is a good candidate for solving this conversion problem. For this paper, a critical depth $Z_{c}$ is defined and is chosen to be $1 \mathrm{mfp}^{\prime}$. Below $Z_{c}$ both diffusion theory and similarity relations can be applied. The $z=Z_{c}$ plane is called the critical plane.

The Monte Carlo simulation step is based on Prahl et al. ${ }^{9}$ Because the implicit photon-capturing technique ${ }^{17}$ is used during the Monte Carlo simulation, a photon packet with an initial weight 1 is launched perpendicular to the surface along the $z$ axis (Fig. 1). If the boundary has the same refractive indices on both sides, all photon weight enters the turbid medium; otherwise, only a portion of the photon weight enters according to Fresnel reflection. Then a step size $s$ is chosen statistically by using $^{9}$

$$
s=-\ln (\xi) /\left(\mu_{a}+\mu_{s}\right),
$$

where $\xi$ is a random number equally distributed between 0 and $1(0<\xi \leq 1)$. The photon packet loses its weight partially at the end of each step as a result of absorption. The amount of weight loss is the photon weight at the beginning of the step multiplied by $(1-a)$, where $a$ is the albedo $\mu_{s} /\left(\mu_{a}+\mu_{s}\right)$. The photon with the remaining weight will be scattered. A new photon direction is statistically determined by the Henyey-Greenstein phase function according to the anisotropy factor $g .{ }^{9}$ Then a new step size is generated by Eq. (1), and the process is repeated.

If the photon packet crosses the surface boundary into the ambient medium, the photon weight contributes to the reflectance $R_{m c}(r)$. If the photon packet reaches a position lower than the critical plane and the photon trajectory points downward (positive $z$-directional cosine) after a new photon direction is statistically determined, then the photon packet is ready to convert into an isotropic photon source. Otherwise, the Monte Carlo simulation continues.

Similarity relation $\mathbf{s}^{15}$ are invoked to convert the photon packet into an isotropic photon source. The similarity relations allow conversion from the anisotropic scatterers into isotropic scatterers with a reduced scattering coefficient $\mu_{s}^{\prime}$ equal to $\mu_{s}(1-g)$, while the absorption coefficient is kept the same. A fixed step of $1 \mathrm{mfp}^{\prime}$, which is the mean free path for the converted isotropic scattering medium, is moved. At the end of this step, the photon packet interacts with the isotropic scattering medium according to an albedo $a^{\prime}$ equal to $\mu_{s}^{\prime} /\left(\mu_{a}+\mu_{s}{ }^{\prime}\right)$. After absorption, the new photon weight becomes the weight at the beginning of the step multiplied by the transport albedo $a^{\prime}$ and will experience scattering. After this scattering event, the photon packet becomes isotropic, and the weight of the photon packet is recorded into the source function $S(r, z)$, which is guaranteed to be zero above the critical plane. Because of the stochastic nature of the Monte Carlo simulation, multiple photon-packet $(N)$ simulations are necessary for meaningful results to be obtained.

Diffusion theory [Eqs. (2)-(9) $]^{7}$ computes the reflectance that is due to an isotropic point source located below the critical plane to yield an impulse response. The recorded source function $S(r, z)$ is taken as the photon source, and these impulse responses weighed by the source function $S(r, z)$ are integrated to obtain the diffuse reflectance $R_{\text {diff }}(r)$.

The diffuse reflectance at $(r, \theta, z=0)$ from a point source at $\left(r^{\prime}, \theta^{\prime}, z^{\prime}\right)$ is

$$
\begin{aligned}
R\left(r-r^{\prime}, \theta-\right. & \left.\theta^{\prime}, z^{\prime}\right) \\
= & \frac{1}{4 \pi}\left[z^{\prime}\left(\mu_{\mathrm{eff}}+\frac{1}{d_{1}}\right) \frac{\exp \left(-\mu_{\mathrm{eff}} d_{1}\right)}{d_{1}^{2}}\right. \\
& \left.+\left(z^{\prime}+2 z_{b}\right)\left(\mu_{\mathrm{eff}}+\frac{1}{d_{2}}\right) \frac{\exp \left(-\mu_{\mathrm{eff}} d_{2}\right)}{d_{2}^{2}}\right]
\end{aligned}
$$

The diffusion theory added an imaging source of the original point source about the imaging plane at $z=-z_{b}$ to satisfy the boundary condition, where $z_{b}$ is

$$
z_{b}=2 A D
$$

where $A$ is related to the internal reflection $r_{i}$. When the boundary has matched refractive indices, $A=1$; other- 
wise, $A$ can be estimated by

$$
A=\left(1+r_{i}\right) /\left(1-r_{i}\right)
$$

where $r_{i}$ is $^{5}$

$$
r_{i}=-1.440 n_{\mathrm{rel}}^{-2}+0.710 n_{\mathrm{rel}}^{-1}+0.668+0.0636 n_{\mathrm{rel}} \text {, }
$$

and $D$ is the diffusion constant

$$
D=1 /\left\{3\left[\mu_{a}+\mu_{s}(1-g)\right]\right\} .
$$

In Eq. (2), $\mu_{\text {eff }}$ is the effective attenuation coefficient

$$
\mu_{\text {eff }}=\left\{3 \mu_{a}\left[\mu_{a}+\mu_{s}(1-g)\right]\right\}^{1 / 2} .
$$

The distance $d_{1}$ between the point of observation at $(r, \theta, z=0)$ and the point source at $\left(r^{\prime}, \theta^{\prime}, z^{\prime}\right)$ is given by

$$
d_{1}=\left[r^{2}+r^{2}-2 r r^{\prime} \cos \left(\theta-\theta^{\prime}\right)+{z^{\prime}}^{2}\right]^{1 / 2},
$$

and the distance $d_{2}$ between the point of observation at $(r, \theta, z=0)$ and the image point source at $\left(r^{\prime}, \theta^{\prime},-z^{\prime}-2 z_{b}\right)$ outside the medium is given by

$$
d_{2}=\left[r^{2}+r^{\prime 2}-2 r r^{\prime} \cos \left(\theta-\theta^{\prime}\right)+\left(z^{\prime}+2 z_{b}\right)^{2}\right]^{1 / 2} .
$$

When the Monte Carlo step of the hybrid simulation is finished, the source function $S(r, z)$ gives the total photon weight in a grid element and may be converted into probability per unit volume $S_{d}(r, z)$, which is called source density. The diffuse reflectance resulting from the distributed source $S_{d}(r, z)$ is calculated by

$$
R_{\mathrm{diff}}(r)=\int_{0}^{\infty} \int_{0}^{\infty} \int_{0}^{2 \pi} S_{d}\left(r^{\prime}, z^{\prime}\right) R\left(r-r^{\prime},-\theta^{\prime}, z^{\prime}\right) r^{\prime} \mathrm{d} \theta^{\prime} \mathrm{d} r^{\prime} \mathrm{d} z^{\prime} .
$$

Because of its cylindrical symmetry, the diffuse reflectance $R_{\text {diff }}(r)$ is independent of the azimuthal angle $\theta$. Therefore we conveniently choose the observation point at $\theta=0$; thus the term $\theta-\theta^{\prime}$ is written $-\theta^{\prime}$ in Eq. (10). The final diffuse reflectance will be the sum of the reflectances computed by the initial Monte Carlo step and the subsequent diffusion-theory step

$$
R_{d}(r)=R_{m c}(r)+R_{\mathrm{diff}}(r)
$$

\section{COMPUTATION}

A grid system is set up on the cylindrical coordinate system for scoring the numerical quantities for this simulation. The grid lines are homogeneously spaced in the $r$ and $z$ directions. The number of grid elements in the $r$ direction and the $z$ direction are $N_{r}^{\prime}$ and $N_{z}^{\prime}$, respectively; and the grid separations along the $r$ and the $z$ coordinates are $\Delta r^{\prime}$ and $\Delta z^{\prime}$, respectively. The center coordinates of the $i$ th $r$ grid element and $j$ th $z$ grid element are $r_{i}{ }^{\prime}$ and $z_{j}^{\prime}$, respectively:

$$
\begin{aligned}
& r_{i}{ }^{\prime}=(i+0.5) \Delta r^{\prime}, \\
& z_{j}{ }^{\prime}=(j+0.5) \Delta z^{\prime} .
\end{aligned}
$$

For this work the grid elements in the $z$ direction need only to be set up for $z \geq Z_{c}$, because all the nonzero sources are in the range $z \geq Z_{c}$. However, we use grid lines for $z \geq 0$ because of simplicity and possible future extension of this model to physical quantities inside the medium.

At the end of the Monte Carlo step, $R_{m c}(r)$ gives the total photon weight reflected into an annulus. We convert the photon weight into reflectance (probability per unit area) by dividing it by the total number of launched photon packets $(N)$ and the area of the annulus $\left(2 \pi r_{i}{ }^{\prime} \Delta r^{\prime}\right)$.

The raw source function $S\left(r_{i}{ }^{\prime}, z_{j}{ }^{\prime}\right)$ is converted into the source-density function $S_{d}\left(r_{i}{ }^{\prime}, z_{j}{ }^{\prime}\right)$ by

$$
S_{d}\left(r_{i}{ }^{\prime}, z_{j}{ }^{\prime}\right)=S\left(r_{i}{ }^{\prime}, z_{j}{ }^{\prime}\right) /\left(\Delta V_{i} N\right)
$$

where $\Delta V_{i}$ is the grid volume confined by the $i$ th $r$ grid element (an annulus) and the $j$ th $z$ grid element,

$$
\Delta V_{i}=2 \pi r_{i}{ }^{\prime} \Delta r^{\prime} \Delta z^{\prime}
$$

Substituting Eq. (15) into Eq. (14), one obtains

$$
S_{d}\left(r_{i}{ }^{\prime}, z_{j}{ }^{\prime}\right)=S\left(r_{i}{ }^{\prime}, z_{j}{ }^{\prime}\right) /\left(2 \pi r_{i}{ }^{\prime} \Delta r^{\prime} \Delta z^{\prime} N\right)
$$

The grid system used to score the source term $S(r, z)$ is used also to compute the integration over $r^{\prime}$ and $z^{\prime}$ in Eq. (10). The symmetry of the integration over $\theta^{\prime}$ is used to lower the upper limit from $2 \pi$ to $\pi$. Therefore $R_{\text {diff }}(r)$ is computed by the following equation, in terms of the source density $S_{d}(r, z)$ :

$$
\begin{aligned}
R_{\mathrm{diff}}(r)= & \sum_{i=0}^{N_{r^{\prime}-2}} \sum_{j=0}^{N_{z}^{\prime}-2} S_{d}\left(r_{i}{ }^{\prime}, z_{j}{ }^{\prime}\right) r_{i}{ }^{\prime} \Delta r^{\prime} \Delta z^{\prime} 2 \\
& \times \int_{0}^{\pi} R\left(r-r_{i}{ }^{\prime},-\theta^{\prime}, z_{j}{ }^{\prime}\right) \mathrm{d} \theta^{\prime} .
\end{aligned}
$$

Note that the last grid elements in each direction are not used in the summation. Only a limited number of grid elements in each direction can be specified, but the step size for the Monte Carlo step has no bound because of the logarithmic operation [Eq. (1)]; therefore the location of a photon packet may extend beyond the grid system. In this case we score the quantities into the last grid element in the direction of the overflow ${ }^{18}$ but do not use the last grid elements in the reflectance computation in Eq. (17). This overflow gives an error that is negligibly small if the grid system is sufficiently large.

Substituting Eq. (16) into Eq. (17), one obtains

$$
R_{\text {diff }}(r)=\sum_{i=0}^{N_{r}{ }^{\prime}-2} \sum_{j=0}^{N_{z}{ }^{\prime}-2} S\left(r_{i}{ }^{\prime}, z_{j}{ }^{\prime}\right) /(\pi N) \int_{0}^{\pi} R\left(r-r_{i}{ }^{\prime},-\theta^{\prime}, z_{j}{ }^{\prime}\right) \mathrm{d} \theta^{\prime} .
$$

Equation (18) indicates that we do not need to convert the source function $S(r, z)$ to the source-density function $S_{d}(r, z)$ for use in Eq. (17). The use of $S(r, z)$ in Eq. (18) is sufficient, which saves computational time.

The integration over $\theta^{\prime}$ in Eq. (18) was done with Gaussian quadratures, ${ }^{19}$ where only 10 function evaluations are required. The sum of $R_{\text {diff }}(r)$ and $R_{m c}(r)$ gives the final diffuse reflectance $R_{d}(r)$.

Both the pure Monte Carlo simulation and the hybrid model are implemented in American National Standards Institute (ANSI) Standard C, which makes the program portable to any machine that supports ANSI Standard C. The pure Monte Carlo simulation program has been suc- 


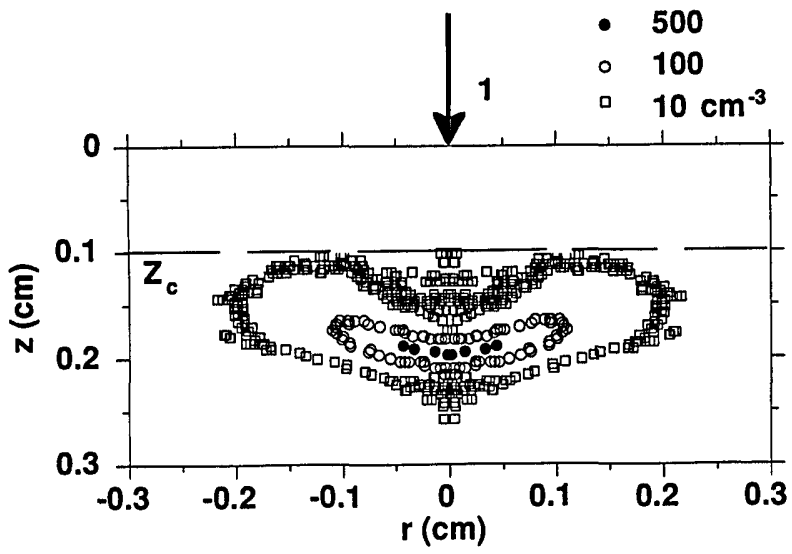

Fig. 2. Contour plot of the source term $S_{d}(r, z)$ (in $\mathrm{cm}^{-3}$ ) for diffusion theory after the Monte Carlo simulation step of 100,000 photon packets in the hybrid model. The optical parameters of the medium are $\mu_{a}=1 \mathrm{~cm}^{-1}, \mu_{s}=100 \mathrm{~cm}^{-1}, \mathrm{~g}=0.9, n_{\text {rel }}=1$. The grid separations for the hybrid model in the $r$ and the $z$ directions are $5 \times 10^{-3} \mathrm{~cm}$ and $3 \times 10^{-3} \mathrm{~cm}$, respectively; and the number of grid elements is 100 in both directions. Note that we intentionally plot versus $r$ in both directions symmetrically, although $r$ is always greater than or equal to zero in the coordinate system.

cessfully tested on Macintoshes, IBM PC and compatibles, and three types of workstation: Sun SPARCstation 2, Silicon Graphics IRIS workstations, and IBM RISC/6000 POWERstation 320 . The pure Monte Carlo simulation program for multilayered turbid media and the corresponding convolution program can be obtained by contacting the authors of this paper.

\section{RESULTS AND DISCUSSION}

An example of the source term $S_{d}(r, z)$ is shown in Fig. 2. The critical depth was chosen to be $1 \mathrm{mfp}^{\prime}$, where $1 \mathrm{mfp}^{\prime}$ is approximately $9.9 \times 10^{-2} \mathrm{~cm}$ in this case. Most of the source strength is concentrated in the neighborhood of $z=2 \mathrm{mfp}^{\prime}$, whereas the overall pattern is heart shaped. The grid separations in the $r$ and the $z$ directions were $5 \times 10^{-3} \mathrm{~cm}$ and $3 \times 10^{-3} \mathrm{~cm}$, respectively, and the number of grid elements was 100 in both directions. This grid system turned out to be sufficient in size because the source terms in the elements around the edge of the grid system were negligible.

An example of the final diffuse reflectance $R_{d}(r)$ is shown in Fig. 3, where the boundary has matched refractive indices. The results from the pure Monte Carlo simulation and the hybrid model agree. If 100,000 photon packets are used in both models, the hybrid model for the medium with this set of optical parameters is about 7 times faster than the pure Monte Carlo simulation. The solid curve with open circles is the diffuse reflectance scored during the Monte Carlo step of the hybrid model $R_{m c}(r)$, which is substantially lower than the final diffuse reflectance $R_{d}(r)=R_{m c}(r)+R_{\text {diff }}(r)$. The comparison between $R_{m c}(r)$ and $R_{d}(r)$ illustrates the contributions of each step of the hybrid model.

To gain the maximum speed advantage of the hybrid model without unacceptable error, we want to stop the Monte Carlo simulation step of the hybrid model as soon as possible, which can be accomplished by choosing a shallow critical depth $Z_{c}$, because photons will reach the criti- cal depth and be converted into isotropic point sources sooner. However, the shallower the $Z_{c}$, the poorer the diffusion approximation; therefore there is a trade-off in the choice of the critical depth. It is obvious that the deeper the critical depth $Z_{c}$, the larger the contribution to the diffuse reflectance from the Monte Carlo step. If $Z_{c}$ is infinite, the hybrid model reduces to a pure Monte Carlo simulation, because all photons will be either absorbed or reflected during the Monte Carlo simulation step of the hybrid model, and the source term $S(r, z)$ will be zero; hence the diffusion step will contribute nothing to the final reflectance.

The computational time of three-dimensional integration is not negligible and can be shortened if the integration in Eq. (10) over the $r^{\prime}$ and the $z^{\prime}$ coordinates is implemented by using the extended trapezoidal method ${ }^{19}$ instead of by summing over the original grid elements, as in Eq. (17). This approach has been used for convolution over photon beams from the impulse response for an infinitely narrow beam, ${ }^{18}$ but for this hybrid model it remains to be investigated. As an example of the computational time dedicated to each step of the hybrid model, the ratio between the time for computing the three-dimensional integral and the time for computing the Monte Carlo simulation step of the hybrid model is approximately 0.57 when we compute the diffuse reflectance with optical parameters $\mu_{a}=1 \mathrm{~cm}^{-1}, \mu_{s}=100 \mathrm{~cm}^{-1}, g=0.9$, and $n_{\text {rel }}=1$ and with 100,000 photon packets. The speed advantage can be enhanched further when only several observation points need to be evaluated for diffuse reflectance, which will save computational time in the diffusion-theory step. For example, the "optimeter" built by Wilson et al. ${ }^{20}$ to investigate photodynamic sensitizers uses only 10 optical fiber collectors.

The speed of the hybrid model is sensitive to the value of $g$ but not to the absorption and scattering coefficients of the medium, because the average number of steps that photons take to get to the critical depth is the dominant factor affecting speed. The mean step size in the Monte Carlo step of the hybrid model is $1 \mathrm{mfp}$. If the critical depth $Z_{c}$ is set to $1 \mathrm{mfp}^{\prime}$, the mean number of steps for photons to get to $Z_{c}$ deep is related to $1 \mathrm{mfp}^{\prime} / 1 \mathrm{mfp} \approx$ $1 /(1-g)$ when $\mu_{a} \ll \mu_{s}(1-g)$; therefore the speed is

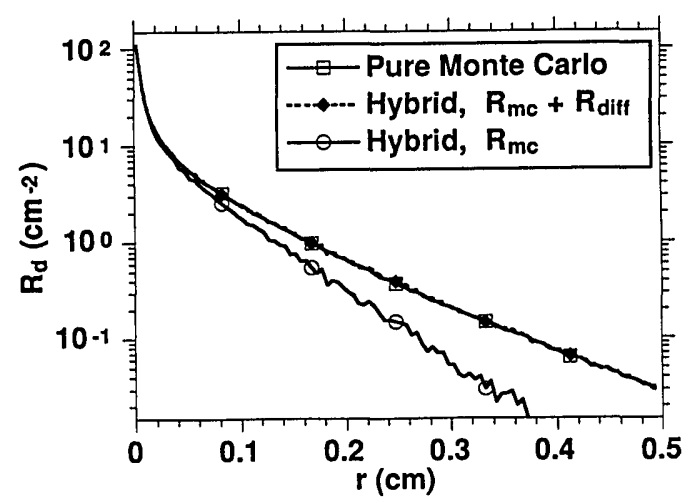

Fig. 3. Comparison between diffuse reflectances from hybrid model and pure Monte Carlo simulation (both with 100,000 photon packets) for an index-matched boundary. The solid curve with open circles is the contribution from the Monte Carlo simulation part of the hybrid model, $R_{m c}(r)$. The optical parameters and the computation settings are described in the caption for Fig. 2. 
independent of absorption and scattering coefficients. To demonstrate this, we conducted hybrid simulations of 100,000 photon packets for two sets of optical parameters: (a) $\mu_{a}=0.1 \mathrm{~cm}^{-1}$ and (b) $\mu_{a}=1 \mathrm{~cm}^{-1}$; and $\mu_{s}=100 \mathrm{~cm}^{-1}$, $g=0.9$, and $n_{\text {rel }}=1$. The two simulations require almost the same amount of computational time. With a larger anisotropy factor $g$ we expect a longer computation, because photons would take more steps to reach the critical depth. The detailed speed profile as a function of optical parameters has yet to be studied.

The speed of pure Monte Carlo simulation is sensitive to absorption and scattering coefficients besides anisotropy. ${ }^{18}$ With the same anisotropy factor $g$, the smaller the absorption coefficient compared with the scattering coefficient, the slower the Monte Carlo simulation, because photons can propagate longer before being terminated. The pure Monte Carlo simulations for the two sets of optical properties in the last paragraph revealed that computation of case (b) $\left(\mu_{a}=1 \mathrm{~cm}^{-1}\right)$ is approximately 2.5 times faster than that of case (a) $\left(\mu_{a}=0.1 \mathrm{~cm}^{-1}\right)$. When the boundary has mismatched refractive indices, the Monte Carlo simulation also is slower compared with that for a medium with matched refractive indices at the boundary, because photons can be reflected back into the medium and travel longer inside the turbid medium.

Therefore, for a given anisotropy, when the ratio between the absorption coefficient and the scattering coefficient decreases, the speed advantage of the hybrid model over the pure Monte Carlo simulation increases. For example, the hybrid simulation for case (a) described above is more than 20 times faster than the pure Monte Carlo simulation. In the case of pure scattering with no absorption, the pure Monte Carlo simulation takes an extremely long time to finish the simulation, because the only way for a photon packet to be terminated is for it to be lost as reflectance; however, some photons can propagate deeply into the medium and take a long time to return. In contrast, the hybrid model propagates photons only until they reach the critical depth $Z_{c}$ and have a downward trajectory.

Because diffusion theory is an approximation and the hybrid model is based on diffusion theory, the hybrid model is an approximation. Therefore we do not expect the hybrid model to produce the same results as the pure Monte Carlo simulation unless the critical depth $Z_{c}$ approaches infinity.

To analyze the difference between the hybrid model and the pure Monte Carlo simulation, we made ten simulations of 100,000 photon packets with both models for a medium with optical parameters $\mu_{a}=1 \mathrm{~cm}^{-1}, \mu_{s}=100 \mathrm{~cm}^{-1}$, $g=0.9$, and $n_{\text {rel }}=1$. The average diffuse reflectances and their standard deviations were then computed. The relative errors for both models are shown in Figs. 4(a) and 4(b). For the pure Monte Carlo simulation, the profile of the relative error increases with the radius $r$, because fewer photons are scored into the grid annulus at larger radius $r$. Although the area of the annulus increases linearly with the radius $r$, the probability of photons being scored into the annulus decreases approximately exponentially for large $r$. In contrast, the relative error of the hybrid model shows no systematic trend. For most points of $r$, the differences between the average diffuse reflectances from the two models are within 1 standard devia- tion of the pure Monte Carlo simulation; and for all points of $r$, the differences are within 2 standard deviations, as shown in Fig. 4(c). The difference between the average diffuse reflectances of the two types of simulations is within $6 \%$ of the average results from the pure Monte Carlo simulations [Fig. 4(d)]. If we want to observe the systematic difference between the two models for a given
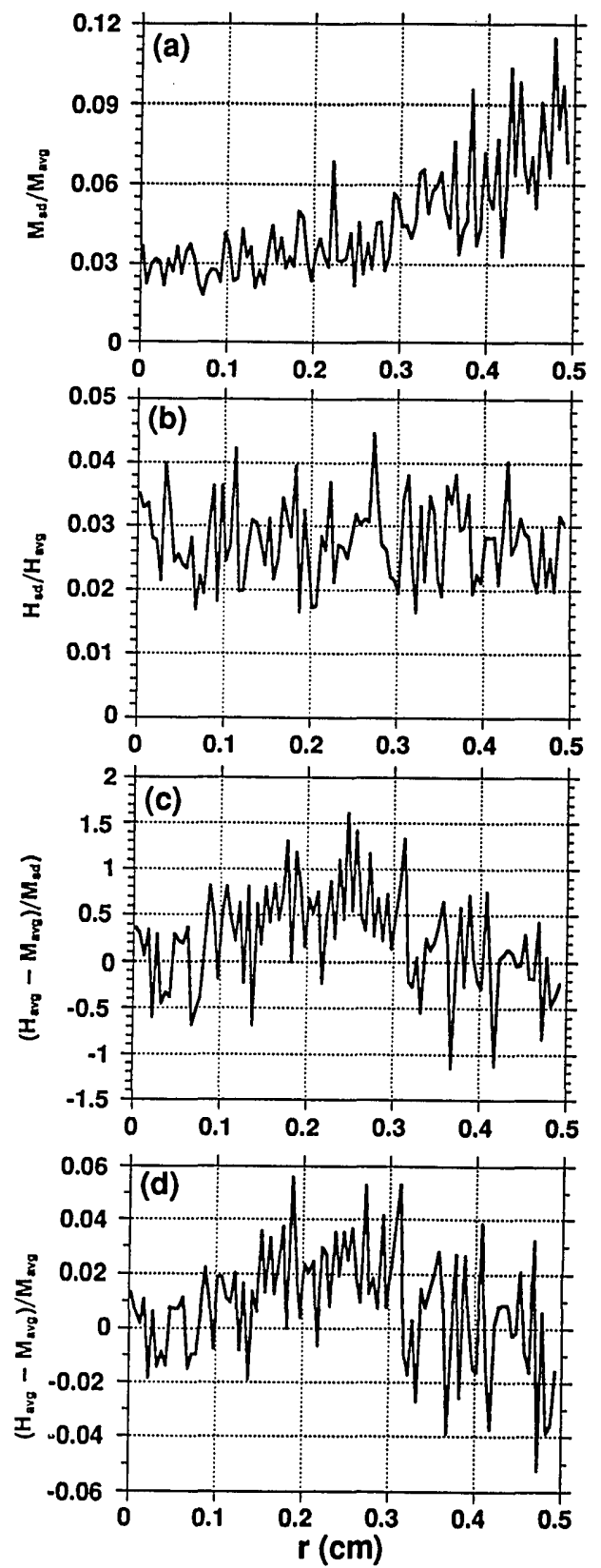

Fig. 4. Error of hybrid model with respect to pure Monte Carlo simulation. Ten runs of 100,000 photon packets were completed for each model. The optical parameters and the grid system are given in the caption for Fig. 2. The average diffuse reflectances of 10 runs are denoted by $M_{\text {avg }}$ and $H_{\text {avg }}$ for the pure Monte Carlo simulation and for that of the hybrid model, respectively, and the corresponding standard deviations are denoted $M_{\text {sd }}$ and $H_{\text {sd }}$. (a) Relative error of the pure Monte Carlo simulation, (b) relative error of the hybrid model, (c) difference between the average values of the hybrid model and of the pure Monte Carlo model divided by the standard deviation of the pure Monte Carlo simulation, (d) difference between the average values of the hybrid model and of the pure Monte Carlo model divided by the average values of the pure Monte Carlo simulation. 


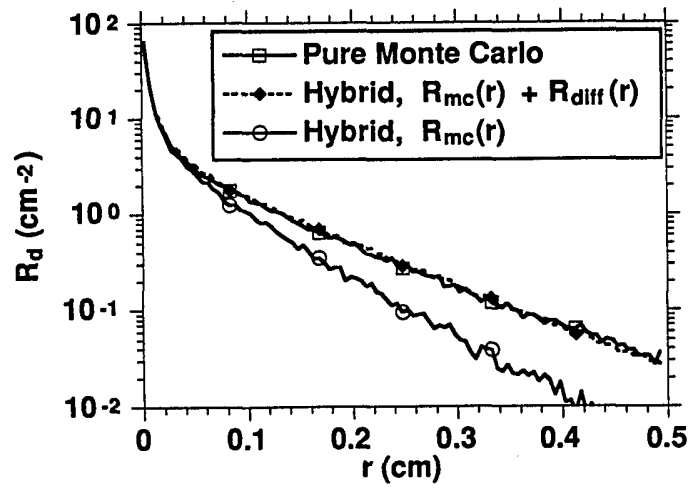

Fig. 5. Comparison of diffuse reflectances from the hybrid model and from the pure Monte Carlo simulation (both with 100,000 photon packets) for an index-mismatched boundary. The solid curve with open circles is the contribution from the Monte Carlo simulation part of the hybrid model, $R_{m c}(r)$. The optical parameters of the medium are $\mu_{a}=1 \mathrm{~cm}^{-1}, \mu_{s}=100 \mathrm{~cm}^{-1}$, $g=0.9$, and $n_{\text {rel }}=1.37$. The grid system is the same as in Fig. 2.

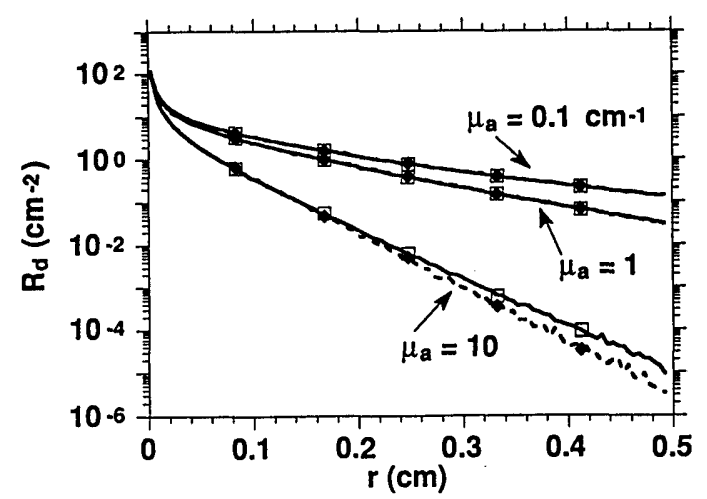

Fig. 6. Comparison of diffuse reflectances from the hybrid model (solid diamonds) and from the pure Monte Carlo simulation (open squares) with varied absorption coefficients while other optical parameters are kept constant $(100,000$ photon packets are used for both simulations). The optical parameters of the medium are $\mu_{s}=100 \mathrm{~cm}^{-1}, g=0.9$, and $n_{\mathrm{rel}}=1$; and varied $\mu_{a}=$ $0.1,1$, and $10 \mathrm{~cm}^{-1}$, respectively. The grid system is the same as in Fig. 2.

critical depth, simulations with substantially more photon packets will be necessary.

Because diffusion theory works for media with boundaries of either matched or mismatched refractive indices, ${ }^{7}$ we expect the hybrid model to work for mismatched boundaries, too. Figure 5 shows an example for a medium with optical parameters $\mu_{a}=1 \mathrm{~cm}^{-1}, \mu_{s}=100 \mathrm{~cm}^{-1}, \mathrm{~g}=$ 0.9 , and $n_{\text {rel }}=1.37$, where $Z_{c}$ still is set to $1 \mathrm{mfp}^{\prime}$ and 100,000 photon packets were computed. The hybrid model still agrees well with the pure Monte Carlo simulation and is about 8 times faster than the pure Monte Carlo simulation.

Diffusion theory is valid only when the absorption coefficient is much less than the reduced scattering coefficient. Because the hybrid model uses diffusion theory, this restriction also applies to the hybrid model. To illustrate this point, with given scattering coefficient $\mu_{s}$, anisotropy factor $g$, and a boundary with matched refractive indices, we computed the diffuse reflectances for three different absorption coefficients by both pure Monte Carlo simulation and by hybrid model (Fig. 6). For $\mu_{a}=$
$0.1 \mathrm{~cm}^{-1}$ and $\mu_{a}=1 \mathrm{~cm}^{-1}$, the two models have a high degree of agreement. However, the hybrid model does not agree with the pure Monte Carlo simulation when $\mu_{a}=$ $10 \mathrm{~cm}^{-1}$, which is comparable to the reduced scattering coefficient $\mu_{s}^{\prime}=10 \mathrm{~cm}^{-1}$.

\section{CONCLUSIONS}

A hybrid model of Monte Carlo simulation and diffusion theory for light reflectance by turbid media has been implemented in Standard C. The hybrid model combines the accuracy advantage of Monte Carlo simulation and the speed accuracy of diffusion theory and is valid when the absorption coefficient is much less than the reduced scattering coefficient, which is exactly where the pure Monte Carlo simulation is slowest. The key to the speed advantage of the hybrid model is to stop the slow Monte Carlo simulation step as soon as possible and let diffusion theory handle the deep photon propagation. The key to the accuracy advantage of the hybrid model is to convert the infinitely narrow photon beam into isotropic photon-point sources deep inside the medium so that diffusion theory can be used with acceptable accuracy.

Although this research considers only the infinitely narrow photon-beam response, the hybrid model can be adapted easily so that it can compute the diffuse reflectance of arbitrary photon sources inside or outside the medium, e.g., a tilted infinitely narrow photon beam or a buried anisotropic photon source. The hybrid model also can be applied to the computation of the fluorescence spectrum where the fluorescence is isotropic by nature. In a word, the hybrid model may be able to solve a large number of practical problems. The technique of transition from the Monte Carlo step to the diffusion-theory step in the hybrid model also should be applicable to similar computations.

\section{ACKNOWLEDGMENTS}

We thank D. Evans of the M. D. Anderson Cancer Center for proofreading the manuscript. This research is supported by the Medical Free Electron Laser Program, Navy contract N00015-91-J-1354 and by National Institutes of Health grant R29-HL45045.

\section{REFERENCES AND NOTES}

1. J. L. Bulnois, "Photophysical processes in recent medical laser developments: a review," Lasers Med. Sci. 1, 47-66 (1986).

2. B. C. Wilson and M. S. Patterson, "The physics of photodynamic therapy," Phys. Med. Biol. 31, 327-360 (1986).

3. M. Keijzer, S. L. Jacques, S. A. Prahl, and A. J. Welch, "Light distributions in artery tissue: Monte Carlo simulations for finite-diameter laser beams," Lasers Surg. Med. 9, 148-154 (1989).

4. S. A. Prahl, "Calculation of light distributions and optical properties of tissue," Ph.D. dissertation (University of Texas at Austin, Austin, Tex., 1988).

5. R. A. J. Groenhuis, J. J. Ten Bosch, and H. A. Ferwerda, "Scattering and absorption of turbid materials determined from reflection measurements. 1: Theory," Appl. Opt. 22, 2456-2462 (1983).

6. R. A. J. Groenhuis, J. J. Ten Bosch, and H. A. Ferwerda, "Scattering and absorption of turbid materials determined from reflection measurements. 2: Measuring method and calibration," Appl. Opt. 22, 2463-2467 (1983). 
7. T. J. Farrell, M. S. Patterson, and B. C. Wilson, "A diffusion theory model of spatially resolved, steady-state diffuse reflectance for the non-invasive determination of tissue optical properties in vivo," Med. Phys. 19, 879-888 (1992).

8. B. C. Wilson and G. Adam, "A Monte Carlo model for the absorption and flux distributions of light in tissue," Med. Phys. 10, 824-830 (1983).

9. S. A. Prahl, M. Keijzer, S. L. Jacques, and A. J. Welch, "A Monte Carlo model of light propagation in tissue," in Dosimetry of Laser Radiation in Medicine and Biology, G. J. Miller and D. H. Sliney, eds., Proc. Soc. Photo-Opt. Instrum. Eng. Institute Ser. IS5, 102-111 (1989). [Note the typo in Eq. (10), where the denominator should be $1-g_{0}+2 g_{0} x$.]

10. A. N. Witt, "Multiple scattering in reflection nebulae I. A Monte Carlo approach," Astrophys. J. Suppl. Ser. 35, 1-6 (1977).

11. S. T. Flock, B. C. Wilson, and M. S. Patterson, "Monte Carlo modeling of light propagation in highly scattering tissuesII. Comparison with measurements in phantoms," IEEE Trans. Biomed. Eng. 36, 1169-1173 (1989).

12. S. T. Flock, B. C. Wilson, and M. S. Patterson, "Hybrid Monte Carlo-diffusion theory modeling of light distributions in tissue," in Laser Interaction with Tissue, M. W. Berns, ed., Proc. Soc. Photo-Opt. Instrum. Eng. 908, 20-28 (1988).

13. S. L. Jacques, C. A. Alter, and S. A. Prahl, "Angular dependence of HeNe laser light scattering by human dermis," Lasers Life Sci. 1, 309-333 (1987).
14. L. G. Henyey and J. L. Greenstein, "Diffuse radiation in the galaxy," Astrophys. J. 93, 70-83 (1941).

15. D. R. Wyman, M. S. Patterson, and B. C. Wilson, "Similarity relations for the interaction parameters in radiation transport," Appl. Opt. 28, 5243-5249 (1989).

16. D. R. Wyman, M. S. Patterson, and B. C. Wilson, "Similarity relations for anisotropic scattering in Monte Carlo simulations of deeply penetrating neutral particles," J. Comput. Phys. 81, 137-150 (1989).

17. H. Kahn and T. E. Harris, "Estimation of particle transmission by random sampling," in Monte Carlo Method, Institute for Numerical Analysis, ed., Natl. Bur. Stand. (US) Appl. Math. Ser. 12 (U.S. Government Printing Office, Washington, D.C., 1951).

18. Lihong Wang and S. L. Jacques, Monte Carlo Modeling of Light Transport in Multi-layered Tissues in Standard C (The University of Texas M.D. Anderson Cancer Center, Houston, Tex., 1992).

19. W. H. Press, B. P. Flannery, S. A. Teukolsky, and W. T. Vetterling, Numerical Recipes in C (Cambridge U. Press, Cambridge, 1988)

20. B. C. Wilson, T. J. Farrell, and M. S. Patterson, "An optical fiber-based diffuse reflectance spectrometer for non-invasive investigation of photodynamic sensitizers in vivo," in Future Directions and Applications in Photodynamic Therapy, C. J. Gomer, ed., Proc. Soc. Photo-Opt. Instrum. Eng. IS6, 219232 (1990). 